\title{
Relationship between national mental health expenditure and quality of care in longer-term psychiatric and social care facilities in Europe: cross-sectional study
}

Tatiana Taylor Salisbury, Helen Killaspy and Michael King

\section{Background}

It is not known whether increased mental health expenditure is associated with better outcomes.

\section{Aims}

To estimate the association between national mental health expenditure and (a) quality of longer-term mental healthcare, (b) service users' ratings of that care in eight European countries.

\section{Method}

National mental health expenditure (per cent of health budget spent on mental health) was calculated from international sources. Multilevel models were developed to assess associations with quality of care and service user experiences of care using ratings of 171 facility managers and 1429 service users.

\section{Results}

Significant positive associations were found between mental health spend and (a) six of seven quality of care domains; and (b) service user autonomy and experiences of care.

\section{Conclusions}

Greater national mental health expenditure was associated with higher quality of care and better service user experience.

\section{Declaration of interest}

None.

\section{Copyright and usage}

(c) The Royal College of Psychiatrists 2017.
In its report entitled Mental Health: New Understanding, New Hope, the World Health Organization (WHO) highlights the need to prioritise mental health and the need to increase expenditure on promotion, prevention and treatment. ${ }^{1}$ More recently, mental health has been included in the Sustainable Development Goals as one of the key health priorities. ${ }^{2}$ Previous research examining the mental health facility expenditure and the quality of care they provide suggest a positive association. ${ }^{3}$ However, it is unclear whether or not greater mental health expenditure at the national level trickles down to better outcomes. The development of the Quality Indicator for Rehabilitative Care (QuIRC), the first internationally standardised tool to assess the quality of care provided in longer-term mental health facilities, ${ }^{4}$ has made it possible to estimate the relationship between national mental health expenditure and quality of care for individuals with longer-term, severe and complex mental health problems. Although a relatively small group, these individuals absorb a high proportion of national mental health budgets because of their need for high levels of support and are, therefore, an ideal population in which to examine this relationship. ${ }^{5}$ Using data collected during the development of the QuIRC, we investigated the association between national mental health expenditure and (a) the quality of care provided in longer-term psychiatric and social care facilities, and (b) service user ratings of the care received, the therapeutic milieu of the facility and their individual autonomy.

\section{Method}

\section{Participants and procedures}

Hospital and community-based residential facilities for people with longer-term mental health problems were purposively sampled in ten European countries as part of the DEMoBinc project. Facilities providing care exclusively to a specific subgroup of service users (for example older people, individuals with intellectual disabilities, patients in forensic settings) were excluded. Facility managers and a random sample of 5-13 service users in each facility participated in face-to-face interviews with a DEMoBinc researcher after providing informed consent to take part in the study. Service users were excluded only if they were not available at the time the researcher was recruiting participants, lacked mental capacity to provide informed consent or were unable to complete the interview. A detailed description of the sampling process is provided by Killaspy and colleagues. ${ }^{4}$ The DEMoBinc project was approved by the relevant ethics committee in each country (see online supplement DS1 for details)

\section{Facility manager ratings of quality of care}

The QuIRC was developed through (a) the synthesis of a systematic review of the evidence for high-quality care, ${ }^{6}$ results from Delphi exercises with service users, carers, advocates and mental health professionals on what helps assist recovery ${ }^{7}$ and national care standards from each of the ten participating countries; and (b) piloting among 213 longer-term psychiatric and social care facilities across ten European countries (Bulgaria, Czech Republic, Germany, Greece, Italy, the Netherlands, Poland, Portugal, Spain and the UK), which took place in $2009 .{ }^{8}$ The QuIRC was validated using service user ratings of care to ensure manager's responses accurately reflected the care provided within the facility.

The instrument is completed by the facility manager and includes 145 items, 86 of which yield percentage scores for the quality of care provided in seven domains: living environment; therapeutic environment; treatments and interventions; selfmanagement and autonomy; social interface; human rights; and 
recovery-based practice (Table DS1). Higher scores indicate better quality care. The instrument was found to have high internal consistency (Cronbach's $\alpha=0.89$ ) and good interrater reliability (average intraclass correlation coefficient $($ ICC $)=0.95){ }^{8}$

\section{Service user ratings of care}

Service users completed standardised assessments of quality of life (Manchester Short Assessment of Quality of Life), ${ }^{9}$ autonomy (Resident Choice Scale), ${ }^{10}$ experiences of care (Your Treatment and Care $)^{11}$ and the therapeutic milieu of the facility (Good Milieu Index $)^{12}$ (Table DS1) in 2009. For all measures, higher scores indicate a more positive experience of care or outcome. Demographic information, including age, gender, diagnosis and date of admission, was sought from the service user and corroborated using case notes.

\section{Mental health expenditure}

National mental health expenditure was estimated as the percentage of the health budget spent on mental health in each country and was used in all analyses. No single source reported expenditure data for all included countries; therefore, estimates were collected from the WHO Mental Health Atlas 2005 (reporting data from 2004) ${ }^{13}$ and data from the Mental Health Economics European Network (MHEEN) ${ }^{14}$ that were based on best available information such as government reports and journal articles. The data include all direct health costs associated with mental health problems such as service utilisation and medication. As Spanish data were reported by region, the average percentage of the health budget spent on mental health across all regions was used as the national statistic. Expenditure information was not available for Greece, therefore, its data were excluded from analyses in this study.

\section{Confounding variables}

Potential confounding variables at both facility and country levels were included a priori, based on the findings of studies previously conducted among this service user group and longer-term mental health treatment settings, ${ }^{6}$ professional opinion and availability of data. Data on facility type (hospital or community), the full-time equivalent (FTE) staff/service user ratio (below or above sample mean) and presence of a maximum length of stay within the facility (yes or no) were collected during the development of the QuIRC. All three variables indicate the goals and expectations of mental healthcare. Country-level variables were limited to publicly available data. Data on stigma perceived by service users with schizophrenia in each country were obtained from Thornicroft and colleagues' paper on the development of the Discrimination and Stigma Scale (DISC). ${ }^{15}$ Data were reported for all included countries except the Czech Republic and consequently, Czech data were excluded from the analyses. The year mental health policies were introduced in each country was obtained from the WHO Mental Health Atlas 2005 country reports to calculate the number of years to 2011 since their introduction.

\section{Data analysis}

Multilevel modelling was used to analyse the data to allow for effects of data clustering at the service user, facility and country levels. For each dependent variable (QuIRC domain ratings and service user rated outcomes), four models incorporating confounding variables at the relevant service user, facility and country levels were developed to examine its association with national mental health expenditure (the independent variable).
A model of best fit was selected for each dependent variable using the corrected Akaike Information Criterion (AICc). ${ }^{16}$ The AIC value represents the difference between the approximated true model and the model that has been developed. The greater the difference between these models, the worse the fit. The AICc was developed to account for small sample sizes. An AICc value was calculated for each of the four models. The model with the lowest value was deemed the best fitting model for the dependent variable. All models were checked to ensure assumptions of normality and homoscedasticity were not violated. Data were analysed using Stata release 12.

\section{Association between quality of care and mental health expenditure}

Four, two-level models were developed to examine the association between quality of care and mental health expenditure, which considered confounding variables at the country and facility levels (online Fig. DS1). In model A, each QuIRC domain was modelled separately as a dependent, facility-level (level 1) variable. National mental health expenditure was entered as an independent, country-level (level 2) variable. In model B, the independent variables facility type and FTE staff/service user ratio were added to model A as facility-level fixed effects. In model C, the degree of national stigma and the number of years since the introduction of mental health policies were added as country-level fixed effects, independent variables to model A. In model D, all facility and country independent variables were added to model A as fixed effects. Variables were added as fixed effects because of the small number of countries (highest level groups) included in the models and the non-random selection of countries and facilities.

\section{Association between service user ratings of care and mental health expenditure}

The association between service user ratings of care was examined in four, three-level models which considered potential confounding variables at the country and facility levels (online Fig. DS2). In model E, each service user rating was modelled separately as a dependent variable at the service user level (level 1). Mental health expenditure was entered into the same model as the independent variable with fixed effect at the country level (level 3). In model F, the independent variables facility type and staff/service user ratio were added as facility-level (level 2) fixed effects. In model G, the degree of national stigma and the number of years since the introduction of mental health policies were added to model $\mathrm{E}$ as level 3 independent, fixed-effect variables. In model $\mathrm{H}$, both facility and country independent variables were added to model $\mathrm{E}$ as fixed effects.

\section{Results}

Managers of 171 longer-term psychiatric and social care facilities and 1429 users of these services from across eight countries involved in developing the QuIRC were interviewed (online Fig. DS3). The majority of facilities were located in the community $(67.2 \%)$ and had a mean of $26($ s.d. $=21)$ beds. A total of 133 $(77.8 \%)$ facilities had no stated maximum length of stay. The mean length of stay was 4.5 years. The average service user was a man $(63.4 \%)$ and 45 years of age. Schizophrenia/other psychosis was the most common diagnosis (71.6\%). National variations in mean QuIRC domain scores and service user ratings of care are presented in Table 1.

Increased national mental health expenditure was found to be significantly associated with all QuIRC domain scores except 


\begin{tabular}{|c|c|c|c|c|c|c|c|c|c|}
\hline & \multicolumn{9}{|c|}{ Country } \\
\hline & Sample & Portugal & Bulgaria & Poland & Italy & Spain & Netherlands & UK & Germany \\
\hline \multicolumn{2}{|l|}{ Health budget spent } & 2.30 & 2.50 & $3.50^{b}$ & $5.00^{b}$ & $5.89^{\mathrm{b}}$ & 7.00 & & $10.14^{b}$ \\
\hline $\begin{array}{l}\text { Per capita mental health } \\
\text { expenditure, Int\$ }\end{array}$ & 117.42 & 37.21 & 7.58 & $22.02^{c}$ & $110.20^{c}$ & $94.65^{c}$ & 182.84 & 198.90 & $285.95^{c}$ \\
\hline \multicolumn{10}{|l|}{$\begin{array}{l}\text { QuIRC domain score, } \\
\text { mean (s.d.) }\end{array}$} \\
\hline $\begin{array}{l}\text { Living environment } \\
\text { Therapeutic }\end{array}$ & $60.92(16.04)$ & $59.18(15.64)$ & 54.10 (18.06) & $49.02(12.87)$ & $64.75(9.57)$ & $46.48(16.81)$ & 70.14 (13.95) & 67.05 (10.75) & 73.81 (7.92) \\
\hline $\begin{array}{l}\text { environment } \\
\text { Self-management }\end{array}$ & $52.15(9.73)$ & $47.82(10.55)$ & $45.56(12.17)$ & $47.47(8.58)$ & $52.60(6.83)$ & $55.72(8.04)$ & $51.58(4.86)$ & $64.52(6.03)$ & $51.78(7.22)$ \\
\hline and autonomy & $55.68(15.80)$ & $49.63(16.47)$ & 44.95 (19.19) & $44.06(9.61)$ & $53.18(9.11)$ & $46.86(10.28)$ & $65.98(9.83)$ & 68.69 (11.03) & 71.85 (8.28) \\
\hline $\begin{array}{l}\text { Social interface } \\
\text { Treatments and }\end{array}$ & $48.59(15.28)$ & $51.96(19.33)$ & $45.76(17.68)$ & 40.09 (14.04) & $49.98(11.85)$ & $59.55(16.38)$ & 47.01 (33.38) & $53.95(12.74)$ & $40.32(11.52)$ \\
\hline interventions & $51.17(9.35)$ & $46.49(10.13)$ & $48.48(11.37)$ & $46.24(7.72)$ & $50.55(6.69)$ & $53.97(9.55)$ & $52.74(7.06)$ & $59.50(8.03)$ & $51.57(8.46)$ \\
\hline $\begin{array}{l}\text { Human rights } \\
\text { Recovery-based }\end{array}$ & $57.36(13.12)$ & $48.70(11.85)$ & $52.36(14.39)$ & $52.97(10.41)$ & $48.11(9.60)$ & $53.73(9.10)$ & $70.78(6.44)$ & $69.7(9.19)$ & $65.74(5.71)$ \\
\hline practice & $52.29(12.81)$ & $44.16(13.41)$ & 45.48 (15.94) & $46.08(10.26)$ & $48.43(8.12)$ & $55.42(8.80)$ & $51.71(8.65)$ & $65.92(9.67)$ & $62.39(8.77)$ \\
\hline \multicolumn{10}{|l|}{$\begin{array}{l}\text { Service user rating } \\
\text { scores, mean (s.d.) }\end{array}$} \\
\hline Quality of life & $4.61(0.89)$ & $4.63(0.87)$ & $4.19(0.89)$ & $4.60(0.85)$ & $4.61(0.75)$ & $4.63(0.94)$ & $4.79(0.89)$ & $4.52(0.86)$ & $4.88(0.89)$ \\
\hline Autonomy & $59.42(12.21)$ & $52.41(11.90)$ & $47.93(9.72)$ & $51.28(7.46)$ & $65.30(7.17)$ & $55.59(10.92)$ & $72.65(7.45)$ & $67.13(8.29)$ & $64.54(7.54)$ \\
\hline Experiences of care & $17.47(4.89)$ & $15.71(4.79)$ & $16.12(4.61)$ & $17.18(5.17)$ & $18.56(4.53)$ & $16.58(4.84)$ & $18.96(4.60)$ & $18.90(5.36)$ & $18.08(4.14)$ \\
\hline Therapeutic milieu & $17.36(4.20)$ & $17.39(4.31)$ & $17.05(4.04)$ & $18.01(4.08)$ & $18.01(4.11)$ & $16.83(4.36)$ & $17.34(4.06)$ & $16.91(4.40)$ & $17.38(4.13)$ \\
\hline
\end{tabular}

social interface (Table 2). Positive significant associations were also found between expenditure and service user ratings of autonomy, quality of life and experiences of care. Mental health expenditure and service user rated therapeutic milieu were not significantly correlated.

In models of best fit, increased national mental health expenditure was associated with higher QuIRC domain scores for living environment (model D, coefficient 1.85, $t=3.26, P \leqslant 0.001$, Table 3 and online Tables DS2-8), therapeutic environment (model $\mathrm{B}$, coefficient $1.46, t=3.16, P \leqslant 0.01$ ), treatments and interventions (model B; coefficient 1.12, $t=3.51, P \leqslant 0.001$ ), self-management and autonomy (model D; coefficient 3.17, $t=6.18, P \leqslant 0.001$ ), human rights (model $\mathrm{D}$, coefficient $2.85, t=3.38, P \leqslant 0.001$ ),

Table 2 Correlation between mental health expenditure and quality and service user ratings of care

\begin{tabular}{|c|c|c|}
\hline & \multicolumn{2}{|c|}{ Pearson correlation, $r$} \\
\hline & $\begin{array}{l}\text { Percentage } \\
\text { mental health } \\
\text { expenditure }\end{array}$ & $\begin{array}{l}\text { Per capita } \\
\text { mental health } \\
\text { expenditure }\end{array}$ \\
\hline Living environment & $0.38^{* * *}$ & $0.17^{*}$ \\
\hline Therapeutic environment & $0.41^{* \star *}$ & $0.18^{* *}$ \\
\hline Self-management and autonomy & $0.60^{\star \star \star *}$ & $0.30^{\star \star \star}$ \\
\hline Social interface & 0.00 & -0.03 \\
\hline Treatments and interventions & $0.34^{\star \star \star}$ & 0.13 \\
\hline Human rights & $0.55^{\star \star *}$ & $0.30^{\star * \star}$ \\
\hline Recovery-based practice & $0.56^{* * *}$ & $0.32^{\star * *}$ \\
\hline Autonomy & $0.51^{* \star *}$ & $0.55^{\star \star *}$ \\
\hline Quality of life & $0.12 * * *$ & $0.15^{\star * *}$ \\
\hline Experiences of care & $0.18^{\star * \star}$ & $0.18^{* * *}$ \\
\hline Therapeutic milieu & -0.03 & -0.02 \\
\hline${ }^{*} P<0.05 ; * * P \leqslant 0.01 ; * * * P \leqslant 0.001$. & & \\
\hline
\end{tabular}

and recovery-based practice (model B, coefficient $2.40, t=7.44$, $P \leqslant 0.001)$. A $1 \%$ increase in the percentage of the health budget spent on mental health was associated with an increase in domain scores ranging from $1.12-3.17 \%$. However, no statistically significant association was found between expenditure and the social interface domain.

Among service user ratings of care, national mental health expenditure was positively associated with autonomy (model $\mathrm{H}$, coefficient 2.27, $t=2.48, P=0.01$; see Table 4 and DS9-12) and experiences of care (model E, coefficient $0.29, t=2.62, P=0.01$ ). However, expenditure was not found to be statistically significantly associated with quality of life or therapeutic milieu. All models of best fit met the assumptions of normality. All models except autonomy were found to have uniform variance of error terms (i.e. homoscedasticity). In order to reduce bias in standard errors, and, as a result, the validity of the models' confidence intervals, three service-user-level outliers were removed.

\section{Discussion}

Expenditure on mental health services, although varied, is largely limited throughout Europe. Previous research found better service user outcomes were associated with greater residential facility expenditure in England, Germany and Italy. ${ }^{17}$ However, evidence of the impact of mental health expenditure at the national level did not exist. This study aimed to address this gap in knowledge. We investigated the relationship between expenditure and quality of care in a large sample of service users who are the most severely affected and resource dependent seen by mental health services. We found greater national expenditure on mental health services was associated with better quality care, greater service user autonomy and more positive service user experiences of care.

Mental health expenditure was not found to be significantly associated with social interface. The social interface domain of the QuIRC includes questions related to service user participation 


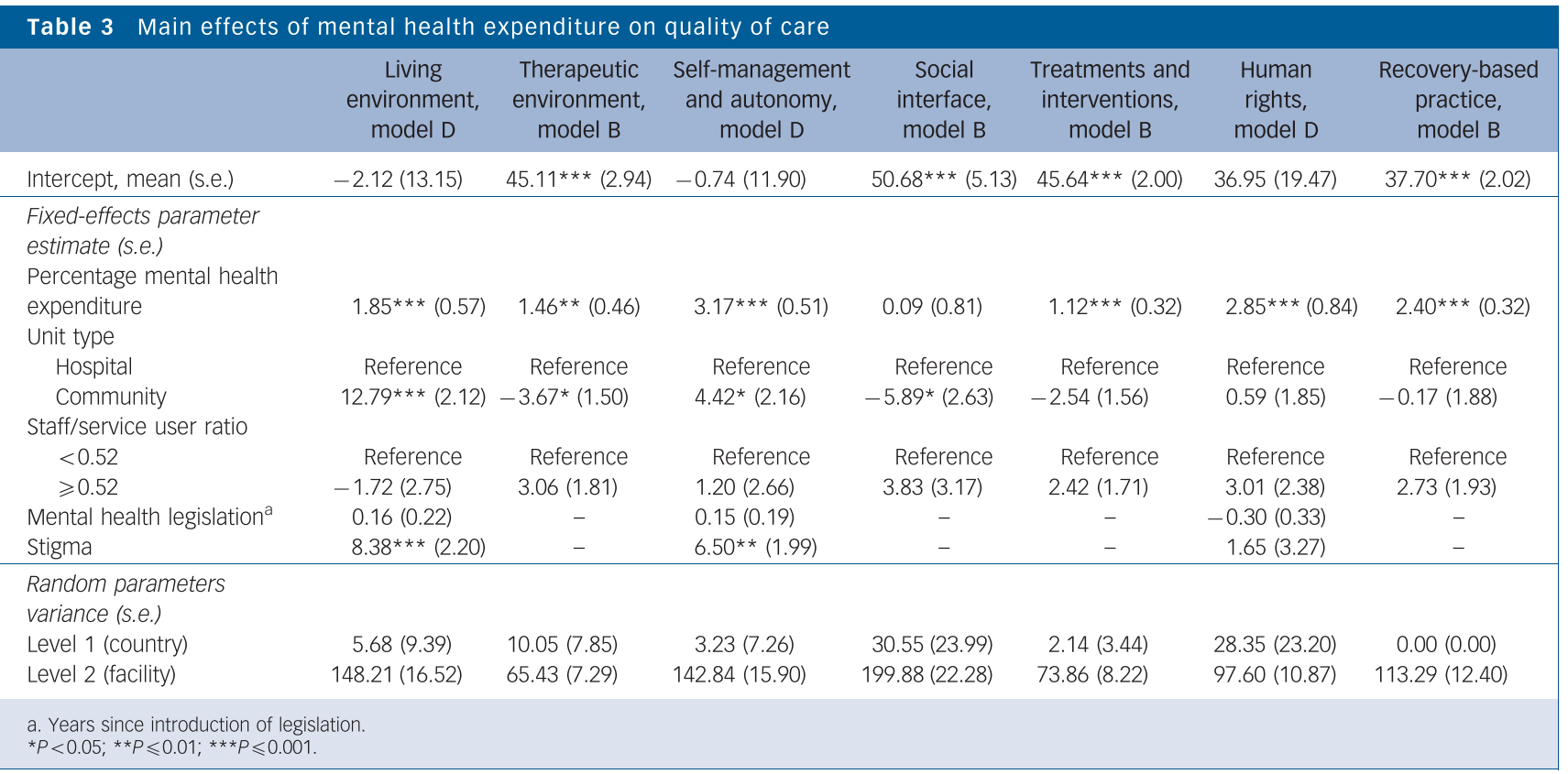

Table 4 Main effects of mental health expenditure on service user ratings of care

\begin{tabular}{|c|c|c|c|c|}
\hline & $\begin{array}{l}\text { Autonomy, } \\
\text { model H }\end{array}$ & $\begin{array}{l}\text { Quality of life, } \\
\text { model E }\end{array}$ & $\begin{array}{c}\text { Experiences of care, } \\
\text { model } E\end{array}$ & $\begin{array}{l}\text { Therapeutic milieu, } \\
\text { model F }\end{array}$ \\
\hline Intercept, mean (s.e.) & $30.72(21.03)$ & $4.40 \star \star \star(0.15)$ & $15.88^{* * *}(0.71)$ & $17.42^{\star \star *}(3.52)$ \\
\hline \multicolumn{5}{|l|}{ Fixed-effects parameter estimate (s.e.) } \\
\hline Percentage mental health expenditure & $2.27 * *(0.92)$ & $0.04(0.02)$ & $0.29 * *(0.11)$ & $-0.08(0.06)$ \\
\hline \multicolumn{5}{|l|}{ Unit type } \\
\hline Hospital & Reference & Reference & Reference & Reference \\
\hline Community & $3.01 *(1.16)$ & - & - & $0.74 *(0.32)$ \\
\hline \multicolumn{5}{|l|}{ Staff/service user ratio } \\
\hline$<0.52$ & Reference & Reference & Reference & Reference \\
\hline$\geqslant 0.52$ & $0.71(1.53)$ & - & - & $-0.15(0.33)$ \\
\hline Mental health legislation ${ }^{a}$ & $-0.11(0.36)$ & - & - & - \\
\hline Stigma & $3.23(3.54)$ & - & - & - \\
\hline \multicolumn{5}{|l|}{ Random parameters variance (s.e.) } \\
\hline Level 1 (country) & $38.67(28.60)$ & $0.03(0.02)$ & $0.54(0.45)$ & $0.02(0.10)$ \\
\hline Level 2 (facility) & $31.24(4.24)$ & $0.05(0.02)$ & $2.70(0.59)$ & $1.28(0.37)$ \\
\hline Level 3 (service user) & $51.34(2.05)$ & $0.71(0.03)$ & $20.07(0.80)$ & $16.28(0.65)$ \\
\hline
\end{tabular}

in activities within the facility and the wider community, staff encouragement and support of service users to engage in activities and the strength of social networks. Facility type was found to have the greatest influence on this domain with service users in hospital settings having higher levels of interaction. This finding seems counter-intuitive given one of the arguments for deinstitutionalisation was increased social integration. However, questions associated with the social interface domain may be more accurately answered by managers of hospital-based facilities who may be better able to monitor service user activities and relationships outside the facility because of the heightened restrictions often placed on service users as compared with those in community settings.

Expenditure was not significantly associated with service user ratings of quality of life or therapeutic milieu. Our inability to find an association between expenditure and service user ratings of quality of life corroborate those of the European Psychiatric Services - Inputs Linked to Outcome Domains and Needs (EPSILON) study that found no association between the cost of psychiatric care and service user life satisfaction in five European countries. ${ }^{18}$ Community-based facilities were significantly associated with higher ratings of therapeutic milieu. Therefore, the amount of money available for care may be less important to this variable than the place where the service user is located.

\section{Limitations}

Mental health expenditure may not have been reported uniformly across the countries included in this study. Expenditure was defined as the proportion of the health budget spent on mental health. However, these figures do not accurately reflect the level of expenditure on mental health in any country as funds often come from several sources including government organisations (for example local authorities, ministries), private insurance and out-of-pocket payments. The types of costs that are included in the health budget also differ by country. For example, some social care costs are included in the mental health budget in the UK, whereas psychotropic medication is subsidised by the Spanish social security system.

Missing expenditure and stigma data resulted in the exclusion of Greek and Czech data, respectively, from this study. The exclusion of Czech data was made as we felt it important to explore stigma associated with mental health problems as a 
potential confounding variable because of its potential to act as a barrier to appropriate mental health funding. ${ }^{19}$ However, as the exclusion of these data accounts for 20 and $29 \%$ of the country and service user sampling frame, respectively, it is important to understand the impact this exclusion has had on the validity of our findings. As a result, we re-ran our models without the stigma variable and an estimate of Greek mental health expenditure of $4.43 \%$ of the health budget as reported in the 2011 version of the Mental Health Atlas. ${ }^{21}$ Although the models of best fit were different for the majority of outcome variables, there were only minor reductions in expenditure coefficient values and no changes in direction or significance levels. We therefore assume that the exclusion of these data did not have a substantial impact on our findings.

Analyses were constrained to facility and service user variables collected as part of the DEMoBinc project and country variables reported in the literature but reflect characteristics relevant to quality and service user ratings of care. The cross-sectional nature of the data made it impossible to investigate potential causal relationships. However, even given comprehensive longitudinal data on service user outcomes, causation may still be difficult to demonstrate given other uncontrolled influences and the possibility that changes in expenditure may not be large enough to have an impact. Despite these limitations, we believe the data from those countries included in this study to be representative of Europe in terms of variations in national wealth and systems of mental healthcare provision. Furthermore, the data are likely to represent the most comprehensive information on quality of longer-term mental healthcare facilities currently available internationally.

\section{Implications}

WHO forecasts predict the burden associated with mental disorders will rise to the second greatest contributor to the global burden of disease, over the next 20 years. ${ }^{21}$ This prediction highlights the need to prioritise and improve the provision of mental healthcare. The results of our study suggest that national mental health expenditure is significantly associated with the quality and service user ratings of mental healthcare. Improved mental well-being not only leads to benefits for service users and their families but has related economic and health benefits for a nation including increased productivity ${ }^{22,23}$ and reduced mental and physical healthcare costs. ${ }^{24}$ Future work in this area should attempt to include a wider array of country, facility and service user variables, as they become available, in order to build more robust models in which the effects of national mental health expenditure might be better understood and service users' outcomes and experiences improved.

\footnotetext{
Tatiana Taylor Salisbury, PhD, Health Service and Population Research Department, Institute of Psychiatry, Psychology and Neuroscience, King's College London, and Department of Population Health, London School of Hygiene and Tropical Medicine, London; Helen Killaspy, PhD, Michael King, PhD, Division of Psychiatry, University College London, London, UK

Correspondence: Tatiana Taylor Salisbury, Health Service and Population Research Department, Institute of Psychiatry, Psychology and Neuroscience, King's College London, De Crespigny Park, London SE5 8AF, UK. Email: tatiana.salisbury@kcl.ac.uk

First received 12 April 2016, final revision 11 Dec 2016, accepted 12 Dec 2016
}

\section{Funding}

This work stems from a study funded by the sixth Framework of the European Commission. The funder had no input in data collection, analysis or manuscript preparation.

\section{Acknowledgements}

DEMoBinc was funded by the Sixth Framework of the European Commission and the authors gratefully acknowledge this support. We also acknowledge the role of the Maristán
Network (http://redmaristan.org/), which facilitated the collaboration of a number of member countries in this study and DEMOBinc partners in collecting facility and service user data.

\section{References}

1 World Health Organization. Mental Health: New Understanding, New Hope. World Health Organization, 2001.

2 United Nations. Sustainable Development Goals 2015. United Nations, 2015 (https://sustainabledevelopment.un.org/?menu = 1300).

3 Knapp M, Hallam A, Beecham J, Baines B. Private, voluntary or public? Comparative cost-effectiveness in community mental healthcare. Policy Polit 1999; 27: 25-41.

4 Killaspy $\mathrm{H}$, King $\mathrm{M}$, Wright $\mathrm{C}$, White $\mathrm{S}$, Mccrone $\mathrm{P}$, Kallert $\mathrm{T}$, et al. Study protocol for the development of a European measure of best practice for people with long term mental health problems in institutional care (DEMoBinc). BMC Psychiatry 2009; 9: 36.

5 Joint Commissioning Panel for Mental Health. Guidance for Commissioners of Rehabilitation Services for People with Complex Mental Health Needs. JCPMH, 2012.

6 Taylor TL, Killaspy H, Wright C, Turton P, White S, Kallert T, et al. A systematic review of the international published literature relating to quality of institutional care for people with longer-term mental health problems. BMC Psychiatry 2009; 9: 55.

7 Turton $\mathrm{P}$, Wright $\mathrm{C}$, Killaspy $\mathrm{H}$, King MB, White S, Taylor T, et al. Promoting recovery in long-term mental health institutional care: an international Delphi study of stakeholder views. Psychiatr Serv 2009; 61: 293-9.

8 Killaspy H, White S, Wright C, Taylor TL, Turton P, Kallert T, et al. Quality of longer term mental health facilities in Europe: validation of the quality indicator for rehabilitative care against service users' views. PLOS One 2012; 7: e38070.

9 Priebe S, Huxley P, Knight S, Evans S. Application and results of the Manchester Short Assessment of Quality of Life (MANSA). Int J Soc Psychiatry 1999; 45: 7-12.

10 Hatton C, Emerson E, Roberts J, Gergory N, Kessissoglou S, Walsh PN. The Resident Choice Scale: a measure to assess opportunities for selfdetermination in residential settings. J Intellect Disabil Res 2004; 48: 103-13.

11 Webb $Y$, Clifford P, Fowler V, Morgan C, Hanson M. Comparing patients' experience of mental health services in England: a five-trust survey. Int $J$ Health Care Qual Assur 2000; 13: 273-81.

12 Røssberg Jl, Friis S. Do the spontaneity and anger and aggression subscales of the ward atmosphere scale form homogeneous dimensions? A crosssectional study of 54 wards for psychotic patients. Acta Psychiatr Scand 2003; 107: 118-23.

13 World Health Organization. Mental Health Atlas 2005. World Health Organization, 2005.

14 Medeiros $H$, McDaid D, Knapp M, the MHEEN group. Shifting Care from Hospital to the Community in Europe: Economic Challenges and Opportunities. PSSRU, 2008.

15 Thornicroft G, Brohan E, Rose D, Sartorius N, Leese M. Global pattern of experienced and anticipated discrimination against people with schizophrenia: a cross-sectional survey. Lancet 2009; 373: 408-15.

16 Akaike H. Factor analysis and AIC. Psychometrika 1987; 52: 317-22.

17 Knapp M, Beecham J, McDaid D, Matosevic T, Smith M. The economic consequences of deinstitutionalisation of mental health services: lessons from a systematic review of European experience. Health Soc Care Community 2011; 19: 113-25.

18 Knapp M, Chisholm D, Leese M, Amaddeo F, Tansella M, Schene A, et al. Comparing patterns and costs of schizophrenia care in five European countries: the EPSILON study. Acta Psychiatr Scand 2002; 105: 42.

19 Sartorius N. Stigma and mental health. Lancet 2007; 370: 810-1.

20 World Health Organization. Mental Health Atlas 2011. World Health Organization, 2011.

21 World Health Organization. Global Burden of Disease: 2004 Update. World Health Organization, 2008.

22 Wells KB, Sherbourne C, Schoenbaum M, Duan N, Meredith L, Unützer J, et al. Impact of disseminating quality improvement programs for depression in managed primary care: a randomized controlled trial. JAMA 2000; 283: 212-20.

23 Rollman BL, Belnap B, Mazumdar S, Houck PR, Zhu F, Gardner W, et al. $A$ randomized trial to improve the quality of treatment for panic and generalized anxiety disorders in primary care. Arch Gen Psychiatry 2005; 62: 1332-41.

24 Chiles JA, Lambert MJ, Hatch AL. The impact of psychological interventions on medical cost offset: a meta-analytic review. Clin Psychol 1999; 6: 204-20. 